\title{
Long-term follow up of patients who were positive for peritoneal lavage cytology: final report from the CCOG0301 study
}

\author{
Yasuhiro Kodera $\cdot$ Seiji Ito $\cdot$ Yoshinari Mochizuki $\cdot$ Norifumi Ohashi $\cdot$ \\ Chie Tanaka $\cdot$ Daisuke Kobayashi $\cdot$ Hiroshi Kojima - Takanori Matsui • \\ Ken Kondo • Michitaka Fujiwara
}

Received: 8 March 2012/Accepted: 17 March 2012/Published online: 21 April 2012

(c) The International Gastric Cancer Association and The Japanese Gastric Cancer Association 2012

\begin{abstract}
Background In gastric cancer patients who have positive results for peritoneal lavage cytology the disease is defined as CY1, and classified as stage IV, and this population has generally suffered a dismal outcome. For this population, we had conducted a phase II trial, with the 2-year survival rate as the primary endpoint, to test the strategy of D2 dissection followed by chemotherapy with single-agent S-1 (1 $\mathrm{M}$ tegafur- $0.4 \mathrm{M}$ gimestat-1 $\mathrm{M}$ otastat potassium). Forty-eight patients were enrolled, of whom 47 were found to have been eligible for analysis. The 2-year survival rate of $46 \%$ exceeded our expectations.
\end{abstract}

For the Chubu Clinical Oncology Group

Y. Kodera $(\bowtie) \cdot$ N. Ohashi · C. Tanaka - D. Kobayashi .

M. Fujiwara

Department of Surgery II, Nagoya University Graduate School

of Medicine, 65 Tsurumai-cho, Showa-ku, Nagoya,

Aichi 466-8550, Japan

e-mail: ykodera@med.nagoya-u.ac.jp

S. Ito

Department of Gastroenterological Surgery, Aichi Cancer Center

Chuo Hospital, 1-1 Kanokoden, Chikusa-ku, Nagoya, Japan

Y. Mochizuki

Department of Surgery, Komaki Municipal Hospital,

1-20 Jobushi, Komaki, Japan

H. Kojima - T. Matsui

Department of Surgery, Aichi Cancer Center Aichi Hospital,

18 Ketsumachi Aza Kuriyado, Okazaki, Japan

K. Kondo

Department of Surgery, Nagoya Medical Center,

4-1-1, Sannomaru, Naka-ku, Nagoya, Aichi, Japan
Methods Further follow up was conducted to confirm whether radical surgery could be recommended for the CY1 population.

Results The 5-year overall and relapse-free survival rates were 26 and $21 \%$, respectively.

Conclusions Gastrectomy with curative intent could be considered for patients with CY1 disease provided they are scheduled to receive effective postoperative chemotherapy.

Keywords Gastric cancer - S-1 - Cytologic examination . Peritoneal carcinomatosis

\section{Introduction}

Stage IV gastric cancer is generally considered incurable and this population is usually ineligible for radical surgery. Treatment options for this population, recommended by the Japanese Guidelines, are chemotherapy, radiotherapy, palliative surgery and palliative care medicine [1]. However, several case series suggest that the possibility of cure cannot be ignored in some carefully selected populations of stage IV patients, given the improvements in multimodal treatment [2-4]. Patients with free cancer cells in the peritoneal cavity could constitute such a population. Detection of free cancer cells by peritoneal lavage cytology predicts the risk of peritoneal carcinomatosis with high specificity [5]. When cancer cells are detected, the positive cytology status is designated as CY1 by the Japanese classification of gastric carcinoma, 2nd English edition [6]. Patients with CY1 status are classified as stage IV even in the absence of macroscopic evidence of peritoneal seeding. Whether this population should be treated radically or palliatively has been an issue for debate [7]. 
The outcome in CY1 patients has been reported to be poor in the East as well as in the West [8, 9], but the recent introduction of novel anticancer agents has changed the picture to some extent. We conducted a phase II trial, named CCOG0301, exploring D2 dissection followed by treatment with $1 \mathrm{M}$ tegafur-0.4 M gimestat-1 M otastat potassium (S-1), in which 48 patients were registered, of whom 47 were eligible for analysis, and we achieved a two-year survival rate of $46 \%$, which exceeded the initial expectations [4]. The choice of a sample size of 50 patients in this study had been based on the hypothesis that the twoyear survival rate would be $36 \%$ and the lower limit of the $90 \%$ confidence interval would exceed $23.5 \%$, which was the upper limit of the $90 \%$ confidence interval for the historical controls whose two-year survival rate had been $13.3 \%$. While this phase II trial was ongoing, a pivotal phase III trial comparing postoperative adjuvant S-1 monotherapy with treatment by surgery alone in patients with stage II/III gastric cancer turned out to show positive results for the S-1 monotherapy arm. Moreover, the incidence of relapse, as peritoneal carcinomatosis, was found to be significantly lower in the $S-1$ monotherapy group [10]. This finding suggests that $\mathrm{S}-1$ is effective against microscopic residual disease in the peritoneal cavity that is undetectable by peritoneal lavage cytology, and also suggests the potential of S-1 to control micrometastases in the peritoneal cavity. Encouraged by these findings, we were motivated to follow the patients for longer, to see what proportion of the patients who were entered in the CCOG0301 trial were actually cured, in order to reconsider the indication for curative surgery in patients with CY1 disease.

\section{Patients and methods}

Forty-seven patients who were registered between February 2002 and July 2006 for the CCOG0301 study underwent further follow up (for a median of 2,337 days or until death) to evaluate the long-term outcome.

Characteristics of the patients who were enrolled in the CCOG0301 study

Eligible patients had to meet all of the following criteria: (1) a confirmed diagnosis of gastric adenocarcinoma and age less than 80 years; (2) gastrectomy with systemic D2 lymphadenectomy performed (splenectomy could be omitted at the discretion of the surgeons); (3) no distant metastasis, with the exception of minimal peritoneal deposits that were completely resected; (4) no prior treatment besides surgery; (5) positive cytologic results for cancer cells on examination of peritoneal washings (CY1); and (6) adequate organ function [4]. Of the 47 eligible patients, 7 patients had peritoneal deposits, which were coresected at surgery. Seven patients were intraoperatively confirmed to have invasion to adjacent organs (T4), and 38 others had serosal invasion. All but five patients were confirmed to have nodal involvement on pathological examination; six patients had metastasis to the paraaortic lymph nodes [4].

\section{Treatment protocol of the CCOG0301 study}

The interval from surgery to the start of therapy was not to exceed 6 weeks. Patients received S-1 at an oral dose of $40 \mathrm{mg}$ per square meter of body-surface area twice daily for 4 weeks, followed by 2 weeks without chemotherapy. Patients with a body-surface area of less than $1.25 \mathrm{~m}^{2}$ received $80 \mathrm{mg}$ daily; those with a body-surface area of $1.25 \mathrm{~m}^{2}$ to less than $1.5 \mathrm{~m}^{2}$ received $100 \mathrm{mg}$ daily; and those with a body surface area of $1.5 \mathrm{~m}^{2}$ or greater received $120 \mathrm{mg}$ daily. This 6-week cycle was repeated in an outpatient setting under medical supervision until disease progression, unacceptable adverse events, or the patient's withdrawal of consent. Adequate dose modification and changes in the treatment schedule were conducted, as described previously [4, 10].

Disease status was assessed once every 3 months on the basis of serum tumor markers and at least once every 6 months by computed tomography (CT) scanning for the first 2 years. Follow-up visits including a CT scan were performed once every 6 months until the patients were considered disease-free at 5 years after the surgery.

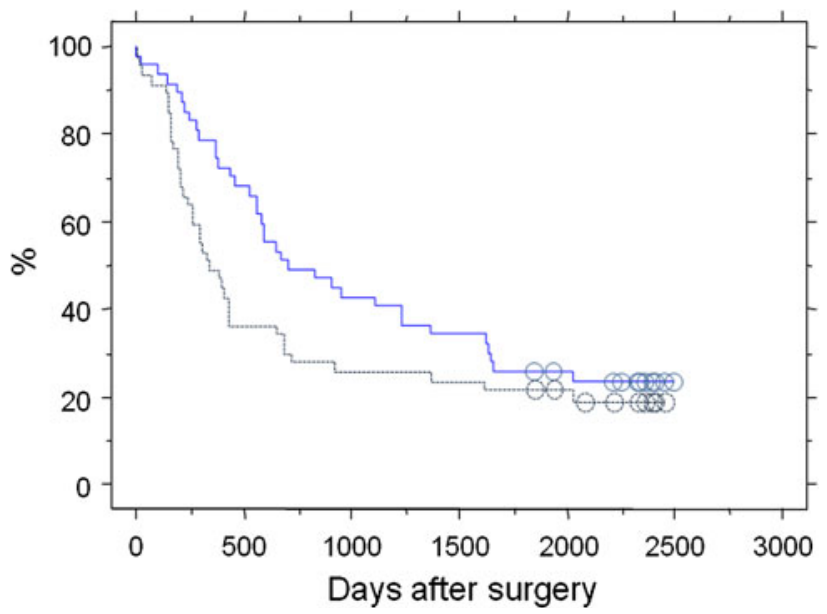

Fig. 1 Overall survival (solid line) and relapse-free survival (dotted line) of patients with gastric cancer who had free cancer cells in the peritoneal cavity and underwent surgery followed by $\mathrm{S}-1$ monotherapy 


\section{Results}

Overall survival and relapse-free survival from the day of surgery are shown in Fig. 1. Median overall survival time was 705 days, and relapse-free survival time was 376 days. The 2- and 5-year survival rates were 46 and $26 \%$, respectively, and the 5-year relapse-free survival rate was $21 \%$. The most frequent pattern of disease recurrence was peritoneal carcinomatosis, occurring in 29 patients $(62 \%$ of all patients enrolled). Other patterns of recurrence were hepatic in 4 patients, lymphatic in 4 , locoregional in 2 , pulmonary in 1 , and osseous in 1 . Three patients died of disease other than gastric cancer, at 5, 1371, and 2023 days after surgery. Details on treatment compliance, dose intensity, and toxicity have been reported previously [4].

\section{Conclusions}

Patients with CY1 disease with no other non-curative factors could be indicated for surgery with curative intent, provided adequate chemotherapy is given. Although postoperative treatment with $\mathrm{S}-1$, now a standard of care for stage II/III gastric cancer, remains as an option, further trials are warranted to decide on the optimal chemotherapeutic regimen and whether to deliver it before or after surgery.

\section{References}

1. Japanese Gastric Cancer Association. Gastric cancer treatment guidelines. 3rd ed. Kanehara: Shuppan (in Japanese); 2010.
2. Sakamoto Y, Sano T, Shimada K, Esaki M, Saka M, Fukagawa T, et al. Favorable indications for hepatectomy in patients with liver metastasis from gastric cancer. J Surg Oncol. 2007;95:534-9.

3. Koga R, Yamamoto J, Ohyama S, Saiura A, Seki M, Seto Y, et al. Liver resection for metastatic gastric cancer: experience with 42 patients including eight long-term survivors. Jpn J Clin Oncol. 2007;37:836-42.

4. Kodera Y, Ito S, Mochizuki Y, Kondo K, Koshikoawa K, Suzuki $\mathrm{N}$, et al. A phase II study of radical surgery followed by postoperative chemotherapy with S-1 for gastric carcinoma with free cancer cells in the peritoneal cavity (CCOG0301 study). Eur J Surg Oncol. 2009;35:1158-63.

5. Leake P-A, Cardoso R, Seevaratnam R, Lourenco L, Helyer L, Mahar A, et al. A systemic review of the accuracy and utility of peritoneal cytology in patients with gastric cancer. Gastric Cancer. 2012 (in press).

6. Japanese Gastric Cancer Association. Japanese Classification of Gastric Carcinoma: 2nd English edition. Gastric Cancer. 1998;1:10-24.

7. Okabe H, Sakai Y. Gastrectomy for patients with positive peritoneal cytology: comments on the article "Positive peritoneal cytology in patients with gastric cancer: natural history and outcome of 291 patients". Ann Surg Oncol. 2011;18:S211-4.

8. Kodera Y, Yamamura Y, Shimizu T, et al. Peritoneal washing cytology: prognostic value of positive findings in patients with gastric carcinoma undergoing a potentially curative resection. J Surg Oncol. 1999;72:60-4.

9. Mezhir JJ, Shah MA, Jacks LM, Brennan MF, Coit DG, Strong VE. Positive peritoneal cytology in patients with gastric cancer: natural history and outcome of 291 patients. Ann Surg Oncol. 2010;17:3173-80.

10. Sasako M, Sakuramoto S, Katai H, Kinoshita T, Furukawa H, Yamaguchi T, et al. Five-year outcomes of a randomized phase III trial comparing adjuvant chemotherapy with $\mathrm{S}-1$ versus surgery alone in stage II or III gastric cancer. J Clin Oncol. 2011;29:4387-93. 